\title{
Desde la capacitación a la articulación. La experiencia de Construcciones 1 en extensión
}

\section{From training to articulation. The experience of Construcciones 1 in extension projects}

\author{
Marcos Cáceres, Martín Justet, Jorge Grisóstolo, Gustavo Pilar y Guillermo Roig \\ Construcciỏnes I, Área de la Tecnología y la Producción, Facultad de Arquitectura y \\ Urbanismo, Universidad Nacional Del Nordeste, Resistencia, Chaco, Argentina.
}

marcos20caceres@gmail.com

\section{RESUMEN:}

La experiencia relatada en este trabajo es el producto de un proceso desarrollado desde hace varios años con diferentes actores e instituciones y enriquecido gracias a las experiencias y las necesidades que surgieron durante el proceso. Las actividades se iniciaron en 2009 como capacitaciones a obreros en municipios del interior del Chaco. Ese proyecto inicial fue ajustándose y creciendo en compromiso a través del programa de extensión "La Universidad en el Medio" y la vinculación con otras instituciones: Facultad de Ingeniería de la U.N.NE. y la U.E.P. №149 de U.O.C.R.A.. Así logramos, en los años sucesivos de implementación, articular la capacitación de los obreros con la formación del alumno de la facultad, promoviendo el intercambio de conocimientos y trabajo, acercando a nuestros estudiantes a la práctica profesional en un proceso continuo de retroalimentación.

\section{ABSTRACT}

The experience reported in this work is the product of a process developed for several years with different actors and institutions and enriched thanks to the experiences and needs that arose during the process. The activities began in 2009 as training for workers in municipalities in the interior of the Chaco. This initial project was adjusting and growing in commitment through the extension program "The University in the Middle" and the link with other institutions: Faculty of Engineering of the U.N.NE. and the U.E.P. UOCRA $N^{\circ} 149$. Thus we managed, in the successive years of implementation, to articulate the training of workers with the training of the faculty student, promoting the exchange of knowledge and work, bringing our students closer to professional practice in a process continuous feedback

PALABRAS CLAVES: proceso, formación, inclusión, retroalimentación.

KEY WORDS: process, training, inclusion, feedback.

FECHA DE RECEPCIÓN: 31/08/2020 | FECHA DE ACEPTACIÓN: 27/10/2020

DOI: http://dx.doi.org/10.30972/arq.0164560 


\section{INTRODUCCIÓN}

En este trabajo presentamos los resultados de actividades prácticas y de extensión realizadas en el marco de Construcciones 1, correspondiente al Área de la Tecnología y la Producción, materia de tercer año de la Carrera de Arquitectura de la Facultad de Arquitectura y Urbanismo de la Universidad Nacional del Nordeste (UNNE).

A través del programa La Universidad en el Medio y de la vinculación con la Facultad de Ingeniería de la UNNE y la UEGP N¹49 de la Fundación Unión Obrera de la Construcción de la República Argentina (UOCRA) hemos logrado dar continuidad a la capacitación de obreros y al intercambio de conocimientos entre éstos y los estudiantes de la UNNE y, al mismo tiempo, favorecer la vinculación entre ambos grupos, como proceso transformador de la realidad social, tendiente a mejorar la calidad de vida y a promover el desarrollo integral y sustentable de los diferentes sectores de la comunidad. Por otra parte, pusimos en contacto a los estudiantes de nuestra universidad con situaciones prácticas y concretas, en las que pudieron aplicar los conocimientos adquiridos, ya que la formación del Arquitecto y del Ingeniero deben promover una estrecha interrelación entre Teoría y Práctica y dotar al profesional de los conocimientos y habilidades -aptitudes y actitudes- requeridos para el ejercicio profesional

El Proyecto, tuvo su origen en una problemática reconocida en nuestro ámbito laboral: la falta de formación práctica del personal que desarrolla tareas en la industria de la construcción. Es una realidad que, en el $90 \%$ de los casos, el primer empleo en la construcción se produce a corta edad, lo que acentúa la exclusión del obrero en el sistema educativo básico, a la que se suma la escasez de formación de los recursos humanos para la actividad de la construcción y afines. Es nuestro propósito, en consecuencia, formar, capacitar y jerarquizar el oficio de esta comunidad. Las actividades se desarrollan dentro de su propio ambiente de formación, la UEGP $N^{\circ} 149$, con el fin de que los asistentes se sientan cómodos en un ámbito conocido por ellos. En este lugar interactuaron los obreros y sus propios docentes, con los alumnos y docentes de la Universidad, modalidad que propicia y garantiza la retroalimentación entre las instituciones y sus actores. Así mismo, en base a esto y dando continuidad al proyecto, planificamos desde la cátedra Construcciones 1 actividades prácticas de campo, en un espacio de aprendizaje extra-áulico dentro del Campus Universitario, como lo es un terreno a cielo abierto y con la realización de trabajos a escala real propiciando la interacción de los alumnos con los docentes de la cátedra, otros profesionales, obreros de la construcción, materiales y herramientas necesarias para la ejecución de los trabajos

Estas actividades permiten identificar las normas de lectura e interpretación correcta y sencilla de los planos para una mayor comprensión de los legajos técnicos, desarrollar un vocabulario adecuado y específico para la obra y mejorar el diálogo y la interacción de los obreros y el profesional que los dirige. Otro aspecto que nos permite esta metodología de trabajo es crear hábitos de actualización permanente. En este campo, presentamos en los encuentros nuevos sistemas tecnológicos utilizados en la región y, gracias a la colaboración de empresarios locales, las clases se realizan con materiales, sistemas y tecnologías innovadoras. La implementación de materiales y técnicas innovadoras favorece el desarrollo de habilidades y competencias a aplicar en situaciones reales.

En cuanto a nuestros alumnos, comprobamos que, a través de estas actividades, pueden mejorar la comprensión de los conceptos teóricos y su consiguiente aplicación en los trabajos prácticos referidos a los planos de un legajo técnico y que los ubica en el ejercicio de la función de Director Técnico de una obra de Arquitectura como así también de Ingeniería.

Las actividades desarrolladas parten de un diagnóstico participativo entre el equipo de la universidad (cátedras, institutos y facultades) a efectos de diseñar la temática a desarrollar en forma participativa, que posibilite ntercambiar y profundizar los temas que se dictan y ejercitan en las clases y trabajos prácticos. 


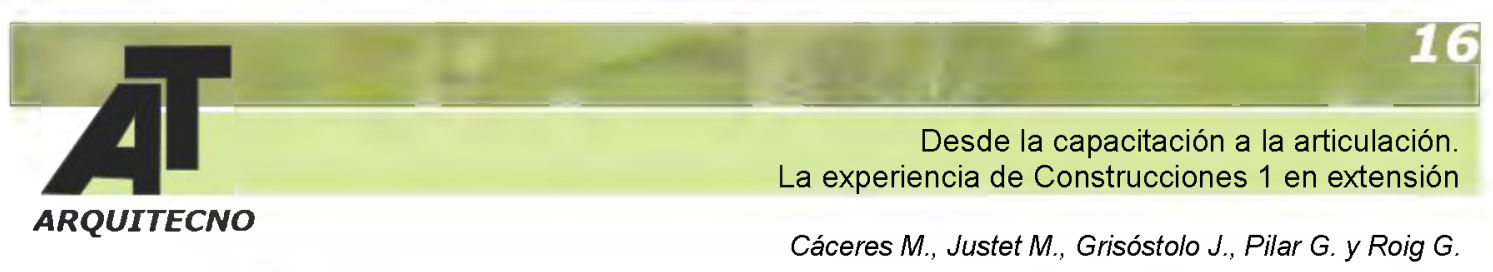

\section{METODOLOGIA}

La primera experiencia, surgida como invitado en un proyecto destinado a reforzar los conocimientos del personal de la construcción, fue intuitiva.

La metodología aplicada es de un aprendizaje participativo implementada en el aporte de conocimientos para la capacitación del personal obrero que cuenta su saber sólo desde lo empírico. Para esto se producen encuentros con los actores, relevando sus necesidades. En cuanto a la participación de los alumnos, la consideramos como una forma de aprendizaje que los vincula con la comunidad, siendo también una Práctica Profesional Asistida, que complementa los conocimientos teóricos con la experiencia de la materialización y la práctica. Por este motivo, desde el inicio de la planificación de temas, actividades y clases incluimos a todos los actores involucrados: estudiantes, obreros, profesores.

La primera relación se plantea con un aporte de conocimiento teórico a la experiencia del personal obrero. La segunda, se da desde la comprensión y la aprehensión de los conceptos desarrollados en las clases teóricas en una situación práctica. Todo esto se integra realizando una experiencia de trabajo práctico de campo con todos los actores juntos, involucrados e interactuando en una misma actividad.

\section{DESARROLLO}

\section{1er Etapa - capacitación de obreros de la construcción}

Esta experiencia se viene realizando en un formato similar desde el año 2015 con la UOCRA sede Resistencia Chaco. En el año 2009 habíamos entablado ya contactos con el municipio de General Pinedo. Durante los años 2010 y 2011 surgió la posibilidad de replicar el Proyecto en la localidad de Presidencia de la Plaza (a $105 \mathrm{~km}$ de Resistencia), siendo esta localidad y Gral. Pinedo, ambas de 2 da categoría, con la finalidad de capacitar cooperativas.

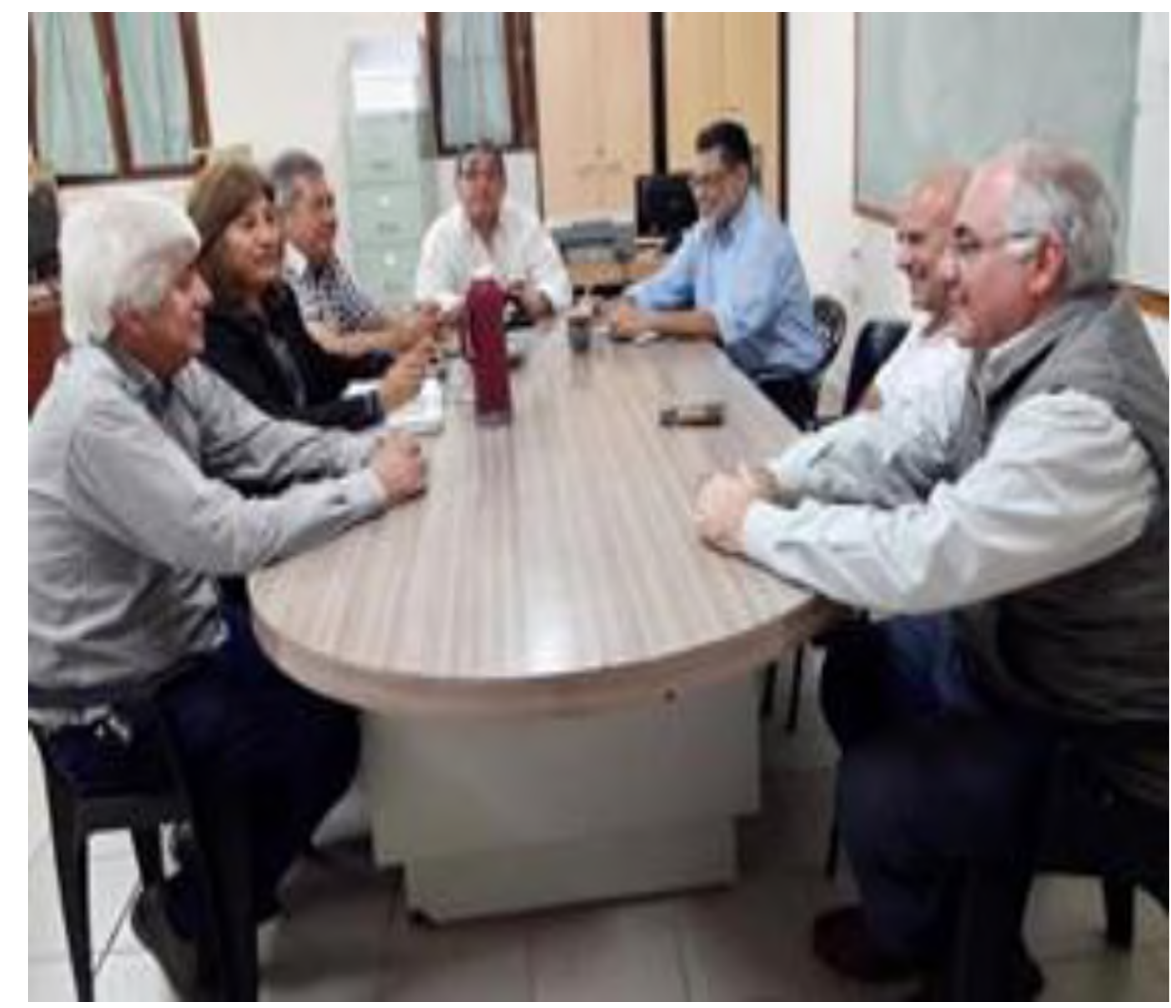

Figura 1: Reunión de las Instituciones involucradas 


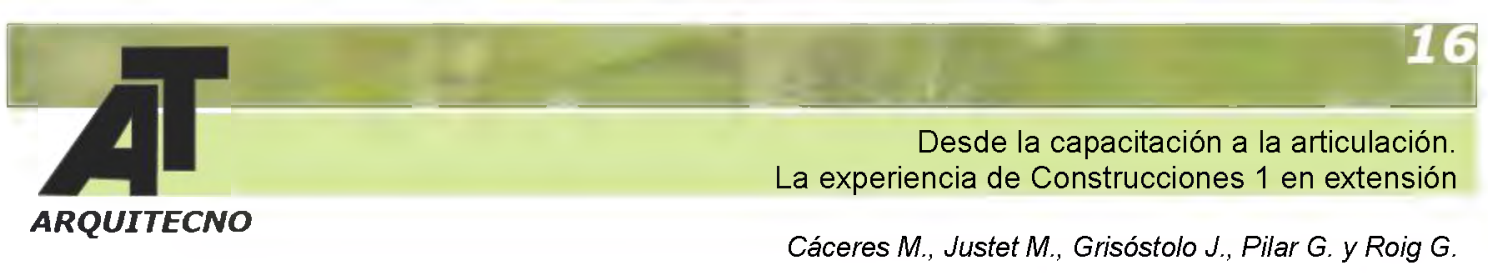

De ahí surgió este proyecto más desafiante, ya que nos encontrábamos a $300 \mathrm{~km}$ de la ciudad de Resistencia.

Desde la cátedra de Construcciones 1 , a partir de la experiencia profesional dentro y fuera del ámbito educativo como de las experiencias previas en el marco de la Extensión Universitaria, hemos advertido que la falta de coordinación entre las instituciones relacionadas a la construcción, la acelerada evolución tecnológica en cuanto a nuevos sistemas y técnicas, junto al déficit que existe en la capacitación de los obreros, hizo que la calidad y cantidad de nuestros oficiales y ayudantes (en sus distintas categorías) haya decaído, llevando a la pérdida de posibilidades laborales. Esta situación se podría modificar formando y capacitando al personal en las nuevas técnicas y sistemas constructivos tanto como diagnosticando malos hábitos laborales en lo que se refiere al buen arte de construir, a la seguridad en las obras, etc.

A través del programa "La Universidad en el Medio" y de la vinculación entre las Facultades de Arquitectura e Ingeniería de la UNNE y el Centro de Extensión de la municipalidad de Gral. Pinedo nos propusimos colaborar en el desarrollo de la capacitación de los obreros, el intercambio de conocimientos, el mejoramiento de la vinculación como procesos transformadores de la realidad social, tendiente a mejorar la calidad de vida y al desarrollo integral y sustentable de los diferentes sectores de la comunidad. Permitir a este sector de la población vulnerable, de empleo informal y cíclico, capacitarse y desarrollarse a fin de lograr una mayor inserción en su actividad laboral y mejorar la valoración de su oficio como obrero de la construcción, fue nuestro objetivo a largo plazo.

En este marco es nuestro propósito formar, capacitar y jerarquizar el oficio de esta comunidad. Las actividades se desarrollan dentro de su propio ambiente de formación.

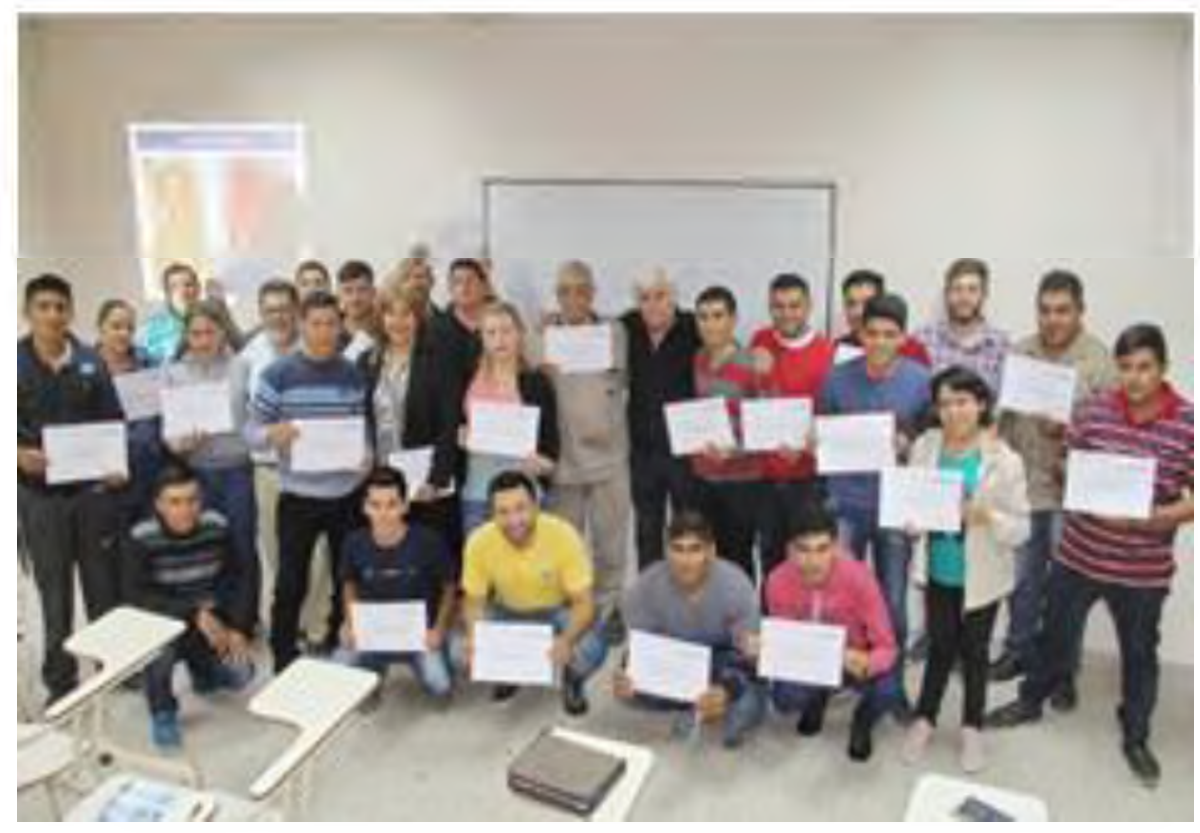

Figura 2: Entrega de certificados a obreros capacitados

Entendemos la educación experiencial como "una estrategia de enseñanza con enfoque holístico, que está destinada a relacionar el aprendizaje académico con la vida real. Con ese fin, propone al alumno realizar actividades en las que, a partir de esa conexión con la práctica, desarrollarán experiencias que resultan de poner a prueba en situación auténtica las habilidades y los conocimientos teóricos que poseen, evaluarán sus consecuencias, enriquecerán esos conocimientos y habilidades e identificarán nuevos problemas." (Camilloni, 2010). El desafío para el educador se convierte claramente en construir metas de contenido, conocimiento, métodos, 


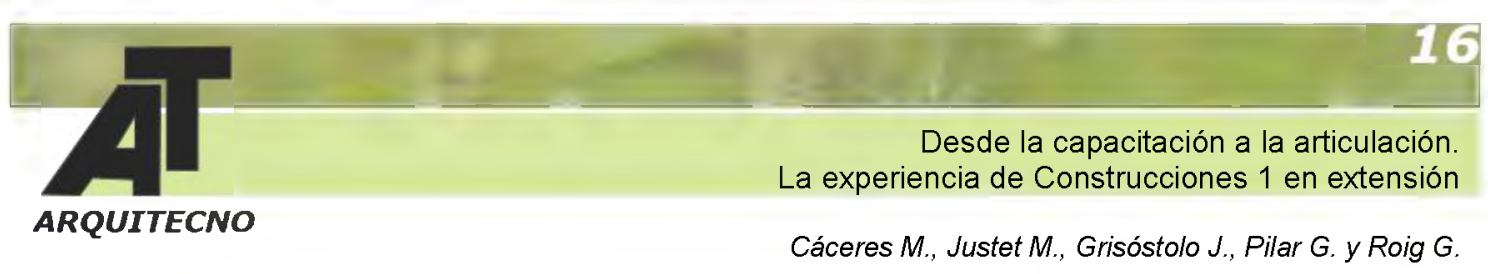

propósitos y formas de comunicación. Los docentes hacen públicas estas metas para los estudiantes y crean mecanismos de valoración que se desarrollan a lo largo del tiempo, puesto que sólo a través de una visión global las comprensiones profundas y flexibles pueden ser desarrolladas.

Un primer aspecto a tener en cuenta dentro de este marco es que la comprensión es un desempeño. La definición de aprendizaje como "apropiación instrumental de la realidad para transformarla", desarrollada en nuestro país por Enrique Pichón Rivière, nos acerca a estos desarrollos más recientes que toman a la comprensión como desempeño: "Para hacer una generalización, reconocemos la comprensión por medio de un criterio de desempeño flexible. La comprensión se presenta cuando la gente puede pensar y actuar con flexibilidad a partir de lo que sabe". En consecuencia, no sólo reconocemos la comprensión mediante un desempeño flexible, sino que podemos afirmar que la comprensión es el desempeño flexible.

Para la articulación básicamente hemos tenido en cuenta las competencias, conocimientos y habilidades en la capacidad de llevar a cabo con eficiencia las tareas pertinentes a la actividad constructiva como un todo, involucrando las técnicas constructivas apropiadas y todas las obras e instalaciones complementarias.

Se desarrollan contenidos como ser la normativa vigente en los códigos de edificación; características de los materiales, técnicas y procesos constructivos, representación técnica en sus distintas escalas y complejidades, el clima y la tecnología, la sustentabilidad.

\section{2da Etapa - formación de Alumnos}

Ante la masividad -400 alumnos en condiciones de asistir a clases- resulta muy complicado llevar adelante un seguimiento personalizado, actividad que resulta imprescindible en una asignatura con contenidos eminentemente prácticos, de aplicación directa.

Para abordar esta problemática nos propusimos involucrar a los alumnos en actividades prácticas supervisadas a los efectos de lograr la comprensión-aprehensión de los conceptos teóricos en ejercicios programados que favorezcan la aplicación práctica de la teoría.

En base a esto, planificamos actividades de campo en un espacio de aprendizaje extra-áulico, como lo es un terreno a cielo abierto y con la realización de trabajos a escala real. Esta metodología la venimos desarrollando en las clases de Construcciones 1 con muy buenos resultados. También hemos involucrado a alumnos de la facultad de Ingeniería para compartir nuestras experiencias.

Gracias a la colaboración de empresarios locales se pudieron realizar clases con la materialización en vivo de estos sistemas y tecnologías. Pretendemos que los estudiantes, con estas actividades, puedan mejorar la comprensión de los conceptos teóricos y su consiguiente aplicación en los trabajos prácticos referidos a los planos de un legajo técnico. 

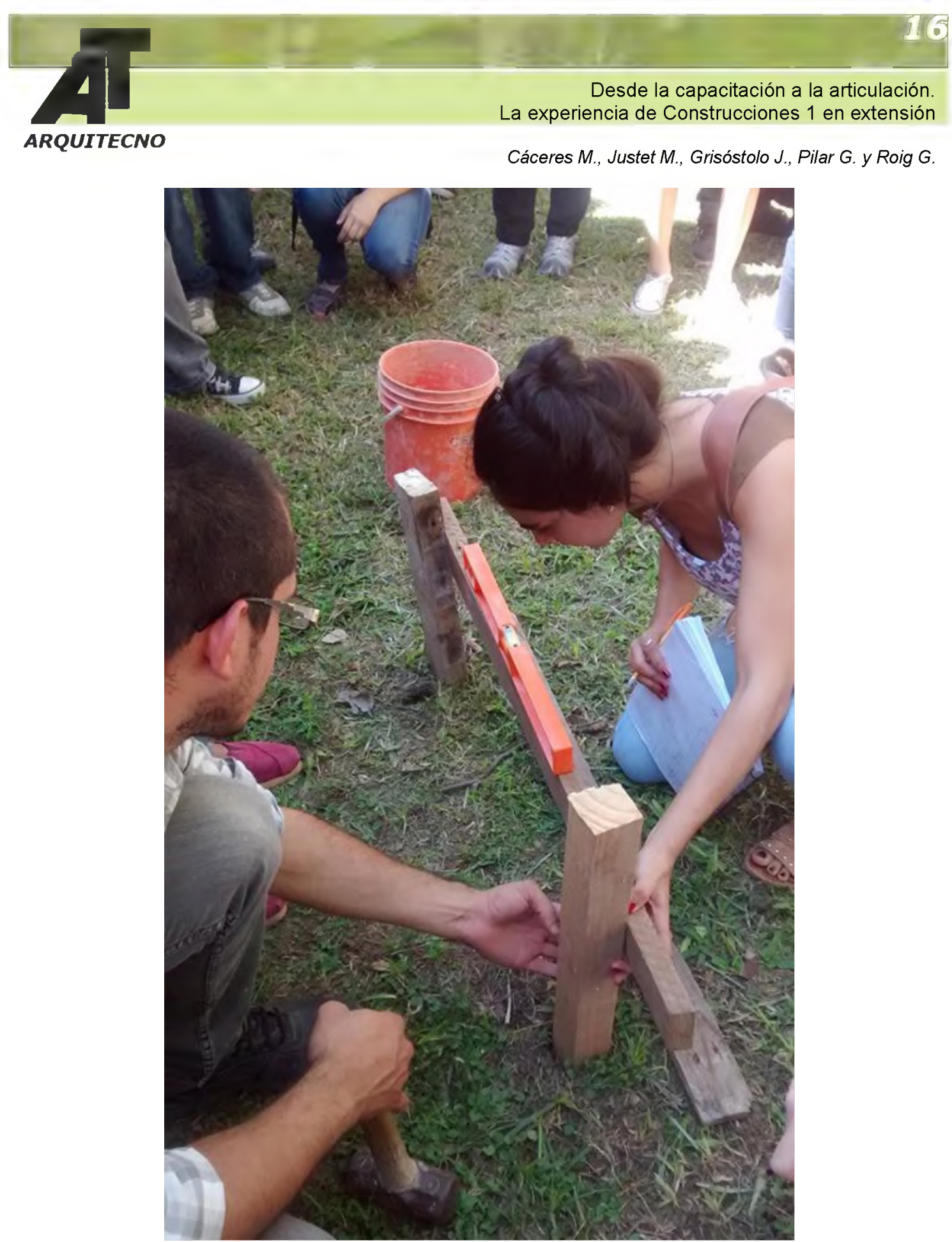

Figura 3: Trabajo Practico de Campo - Docentes Alumnos

Los trabajos prácticos "in situ", contaron con el apoyo docente, transformándose en una tarea "práctica que fue permanentemente guiada", sin que esto signifique una disminución en la participación de los alumnos como protagonistas, ya que fueron ellos los encargados de tomar niveles, sacar escuadras, tomar plomadas, tirar hilos, armar caballetes de replanteo, nivelarlos, y replantear una obra según croquis adjunto desarrollado a tal efecto. 


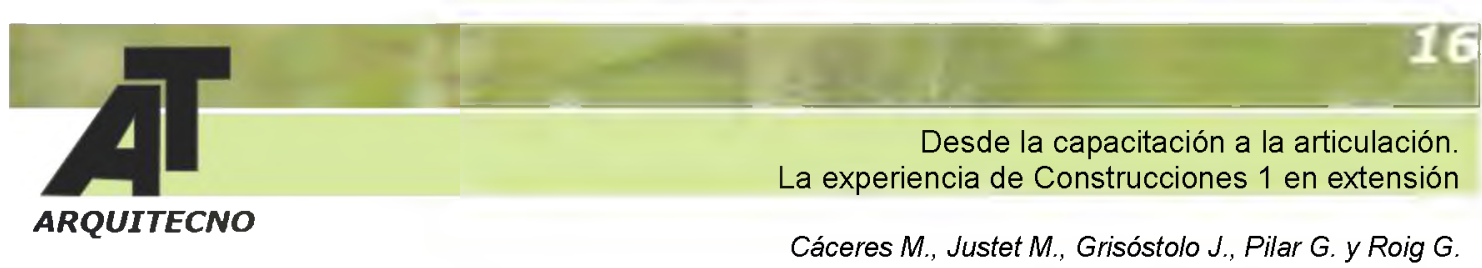

Planificación de las clases:

T.P. N ${ }^{\circ} 1$ Replanteos

Generalidades del práctico:

- Tareas experimentales a desarrollar en grupos de 50 alumnos.

- $\quad$ Actividades realizadas en dos jornadas de 2 horas reloj cada una.

- Modalidad: interacción de docentes, alumnos y oficiales experimentados en el oficio de la construcción.

- $\quad$ Lugar: patio de la Facultad de Arquitectura.

Los pasos con los que se llevó a cabo el práctico son los siguientes:

1. Entrega a los alumnos de material guía para desarrollo del práctico.

2. Determinación de los límites del lote o parcela $(5,00 \mathrm{~m} \times 10,00 \mathrm{~m})$.

3. Obtención del plano de referencia de niveles a $+0.50 \mathrm{~m}$ del terreno natural.

4. Colocación de los ejes de referencia según plano de replanteo. Se reforzó la idea de ubicación y referencias acotadas para poder materializarlo correctamente o con el mejor criterio. 5. Obtención de la escuadra (ángulo de $90^{\circ}$ ) a través del método $3-4-5$ con fundamentación por Pitágoras.

6. Materialización de ejes de referencia (ejes de replanteo) - Ejecución de caballetes Nivelación - Escuadras - Tensado de hilos.

7. Replanteo de Muros según plano. Mamposterías rectas a $90^{\circ}$ - oblicuas a $45^{\circ}$ y curva. Mochetas de puerta y ventana (umbral, antepecho y dintel).

8. Manejo de cotas Progresivas y parciales.

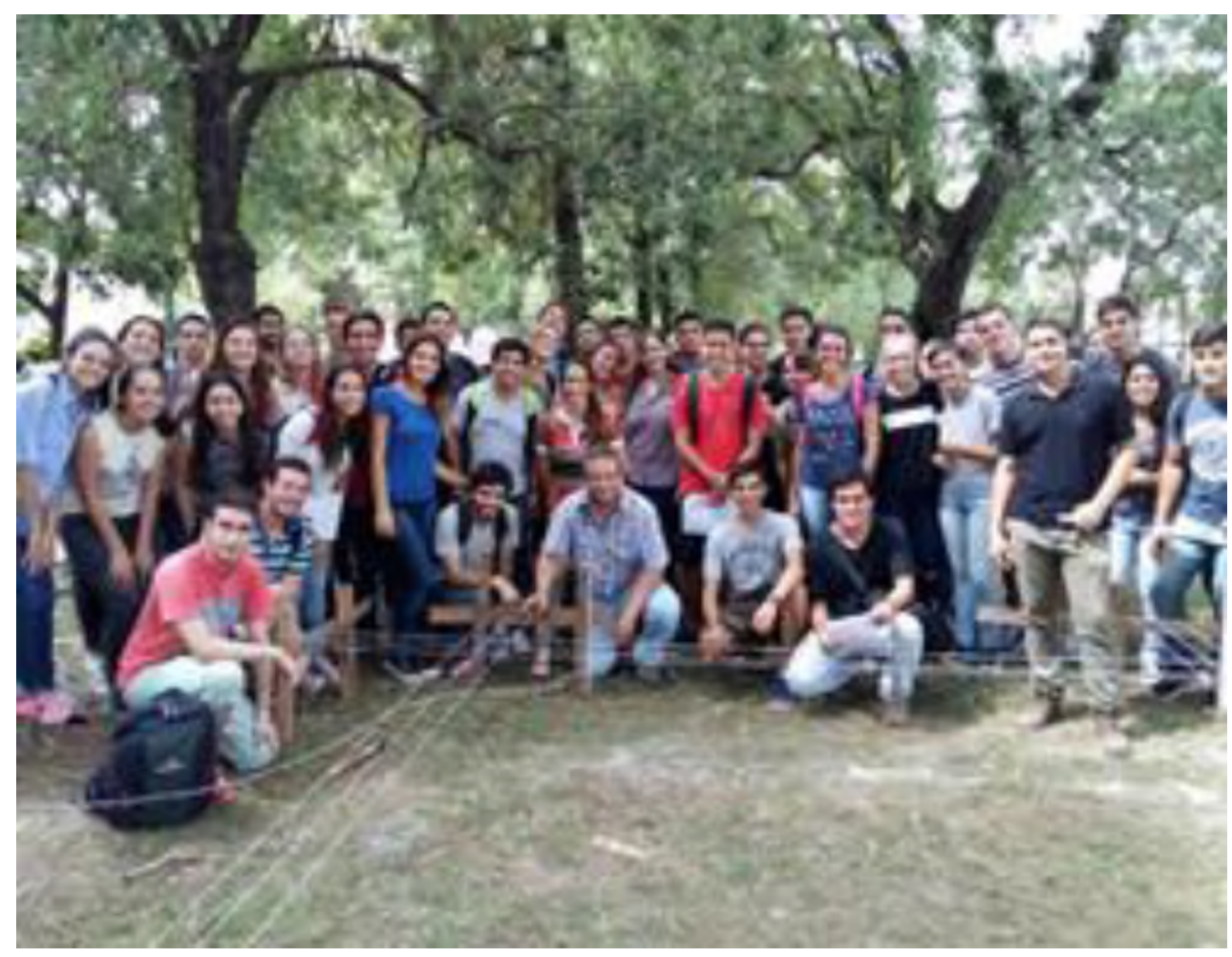

Figura 4: Trabajo de Replanteo finalizado 


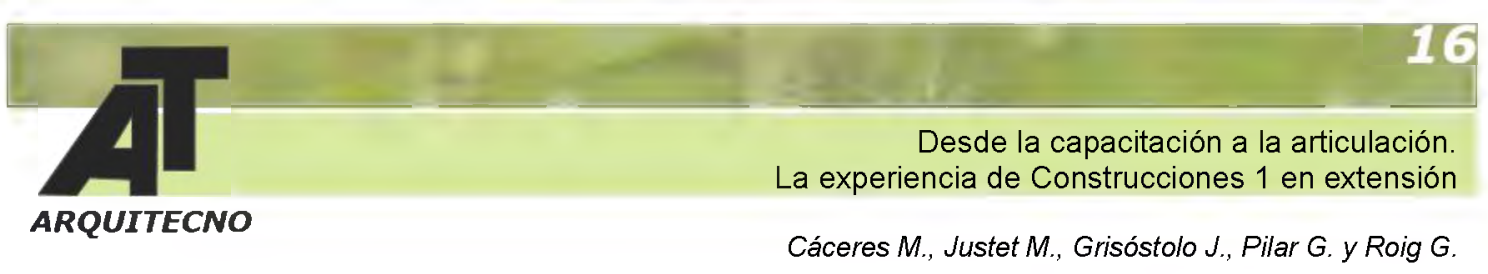

T.P N4 Escaleras

Similar al práctico anterior

- Utilizando las instalaciones del Club Universitario; con el estadio cubierto convertido en aula, se convocó a la totalidad de los alumnos regulares del curso 2013 para otra clase netamente experimental.

- Se materializó un espacio con tecnología de montaje en seco (trabajo "in situ" de dos oficiales instaladores)

- $\quad$ Con la interacción del equipo docente y los alumnos, se realizó la experiencia del replanteo de una escalera, plasmándola en su totalidad en el mismo lugar.

Optamos por realizar este práctico en sólo un día porque los alumnos efectivos eran menos que los inscriptos.

Asimismo, aprovechamos esta instancia para armar un tabique de roca de yeso donde se graficó el perfil de la escalera, por lo que los alumnos pudieron ver la materialización del mismo, para ello contamos con la colaboración desinteresada de la empresa El Galpón Maderas, que ha provisto de materiales y mano de obra.

\section{3er Etapa - integración de Etapas 1 y 2}

A partir del uso de las estadísticas como herramientas de autoevaluación, encontramos de manera muy marcada un bajo grado de comprensión en los trabajos prácticos en general. Hemos buscado una nueva estrategia que refuerce las instancias de aprendizaje existentes (teóricos, prácticos, visita de obra y capacitaciones), implementando entonces, trabajos de campo en los temas de Replanteo y Escaleras con participación directa de alumnos, docentes y personal obrero donde se pone en práctica todo lo abordado desde la clase teórica y de las correcciones programadas.

Como todos los contenidos del programa, se inicia el dictado de la unidad con una clase teórica, apoyada con material gráfico -power point, videos, fotografías, maquetas, etc. Al finalizar el desarrollo teórico se "introduce" al alumno en el trabajo práctico, que consiste en la elaboración de una documentación específica en base a los conceptos abordados. En clases sucesivas se corrigen las producciones parciales. En esta instancia, visualizamos el problema que nos preocupa y ocupa. En este marco, las correcciones grupales y el uso de las nuevas tecnologías se han hecho un soporte indispensable.

Si bien es cierto que el alumnado cuenta en la actualidad con herramientas como la informática y con mucha y variada información al instante a través de internet, también es verdad que muchos no logran integrar y relacionar los contenidos de las diferentes unidades. Como tampoco logran articular por sí solos los diferentes campos del conocimiento. Para conjugar teoría y práctica de manera efectiva nos propusimos desarrollar otra modalidad para la concreción de dichos prácticos.

\section{EJECUCIÓN DE LA PROPUESTA}

A partir del diagnóstico realizado, propusimos una modalidad de clase diferente, con trabajos de campo en dos unidades particularmente significativas: Replanteo y Escaleras

En síntesis, organizamos espacios de aprendizaje extra-áulicos, en terrenos a cielo abierto, a escala real y ejecutamos dos encuentros. En ambos casos, los alumnos asumieron un rol de participación activa. En el primero, fueron, con ayuda de los obreros y docentes, los encargados de tomar niveles, sacar escuadras, verificar verticalidades, tirar hilos, armar caballetes de replanteo, nivelarlos y replantear una obra siguiendo la guía presentada. En el caso del ejercicio para el práctico de escaleras, los estudiantes tuvieron a su cargo la ejecución del replanteo de escaleras, trasladando las dimensiones del plano a escala real, haciendo uso de cinta métrica, niveles láser, nivel de manguera, nivel de mano, plomada y reglas. Realizando traslado de 


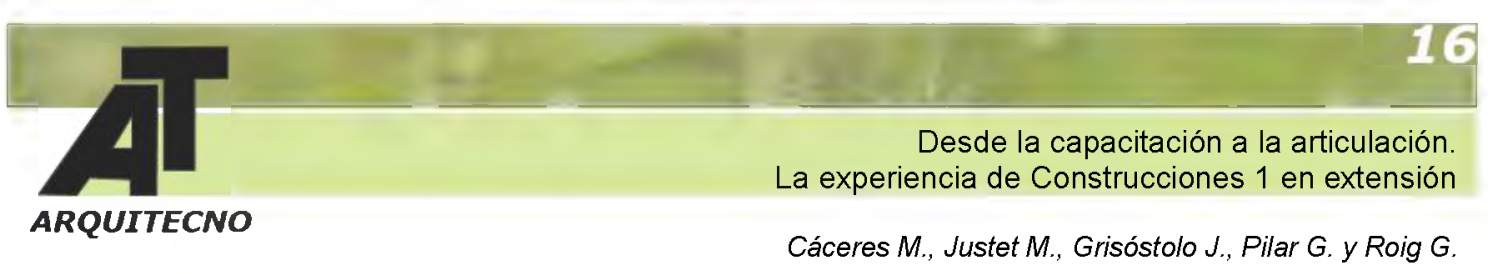

niveles, cambio de planos verticales, dejando la escalera dibujada sobre los paramentos a la espera de la ejecución de un encofrado, tarea esta que no se realiza en esta instancia.

La interacción entre los grupos de alumnos, el equipo docente -conformado por profesionales con una vasta experiencia en el ejercicio independiente- y el personal obrero perteneciente a la UOCRA, permitió una relación más directa y productiva entre alumnos, docentes y trabajadores de la construcción.

Dividimos a los alumnos en 8 grupos, 4 por clase. Los trabajos prácticos "in situ" contaron con el apoyo docente transformándose en una tarea "práctica que fue permanentemente guiada", sin que esto signifique una disminución en la participación de los alumnos como protagonistas, ya que fueron ellos los encargados de ejecutar los trabajos y replantear así un sector de la obra según croquis adjunto confeccionado a tal efecto.

Para lograr la máxima cantidad de alumnos participando activamente de estos prácticos, se les permite realizar una tarea por vez en grupos de 2, 3 o 4 personas dependiendo del trabajo a realizar y con la rotación permanente de los estudiantes que de manera voluntaria se van sumando a las actividades. En estas instancias es posible repetir los procedimientos las veces que sea necesario y allanar todas las dudas que se plantean. En el caso de los replanteos de fundaciones y muros los participantes se subdividen en tres lotes, trabajando de manera simultánea. Si bien, es cierto, que no puede participar el 100\% de los alumnos, no obstante, de esta manera, se logra un alto porcentaje de participación directa y al menos la totalidad de los mismos tiene la posibilidad de ver y escuchar.

Detectamos, cada vez con mayor frecuencia, un alto porcentaje de trabajos insuficientes y de manera más acentuada en Replanteos y Escaleras que luego deben ser recuperados. Las fallas se centran, sobre todo, en que se trata de una situación inédita para el alumno, de práctica profesional, con conceptos nuevos y muy poco tiempo para madurarlos, por lo que buscamos estrategias que disminuyan esa brecha entre la teoría y la práctica.

Esa información con la que contamos al cierre del año lectivo nos permitió detectar falencias en la conceptualización de los temas y por ende la dificultad manifiesta en la comprensión para la correcta realización de los prácticos de Replanteo de fundaciones, muros de planta baja y muros de planta alta. También en trabajos de desarrollo y Replanteo de Escalera y en mayor medida cuando la misma es del tipo compensada.

\section{CONCLUSIONES}

Aportar al desarrollo y la capacitación del personal obrero de la construcción y los formadores de las instituciones que interactúan, mejorando la calidad de vida de las personas en aspectos económicos sociales y comunitarios, a partir de un mejor servicio y gestión en la construcción de las obras de la región, fue nuestro objetivo.

A través de la implementación de estos trabajos de campo, logramos mejorar la comprensión de contenidos difíciles de visualizar solamente de manera teórica, generando un nuevo espacio formativo para los estudiantes universitarios a partir de la práctica.

Como resultados tangibles, a partir de la aplicación de esta metodología de práctico, verificamos una mejora en la cantidad de trabajos prácticos aprobados. Esto se observa a través de los análisis de estadísticas realizadas al final del cursado de la materia.

Como resultados intangibles, percibimos en los alumnos un mayor interés por el cursado y una mejora en lo actitudinal, manifestado claramente en el nivel de asistencia a estas propuestas, así como en las encuestas y en las consultas de los estudiantes hacia el equipo docente.

Las experiencias fueron muy amenas y se desarrollaron en un ámbito de entusiasmo e interés pocas veces visto. La modalidad del trabajo práctico experimental con la participación de los alumnos en forma directa en interacción persona a persona con el equipo docente y los obreros 


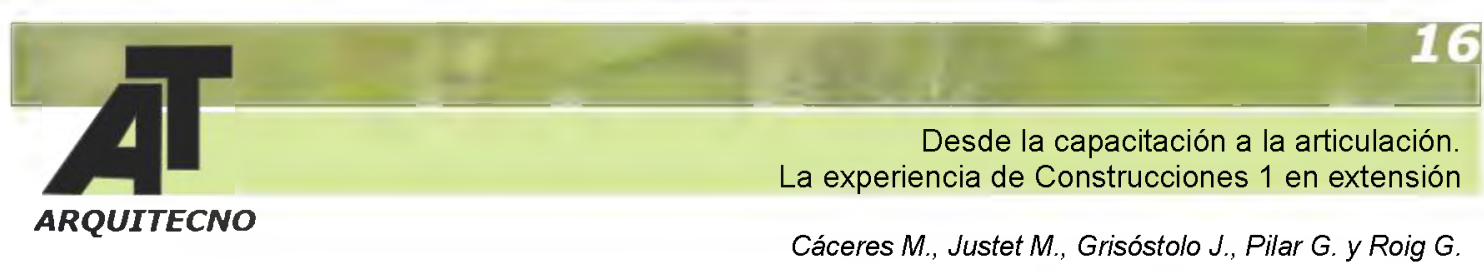

especializados; el manejo de materiales y herramientas dan condiciones únicas para el aprendizaje.

Creemos, como equipo docente, que la realización de estas actividades, en la integración de obreros, alumnos, docentes, instituciones y empresas; aplicadas en el ámbito de la formación, la tecnología constructiva y la producción; es un proceso de transferencia y retroalimentación del conocimiento altamente satisfactoria para todas las partes. Donde por sobre todas las cosas, desde la Universidad Pública, se da la posibilidad de Inclusión de las personas.

\section{BIBLIOGRAFÍA}

Celman, S. (2001). Conferencia inédita plenaria abril, Carrera Especialización en Docencia Universitaria, Resistencia - Chaco. (Inédita)

Dardano de Chandias, M. L. (1987). Diseño y sistemas constructivos de escaleras. Buenos Aires: Alsina.

lucci, C. (2011). "La inclusión de la extensión en la formación de los estudiantes de la Universidad Nacional del Litoral". +E: Revista De Extensión Universitaria, 1(1), 76-78.

Menendez, G. (2013). Integración Docencia y Extensión. Otra forma de enseñar y de aprender. Santa Fe: Universidad Nacional del Litoral.

Nieto, N. (2011). Construcción de edificios. Buenos Aires: Editorial Nobuko.

Pasman, M. F. (1951) Materiales de Construccion. Buenos Aires: Cesarini Hermanos.

Perkins, D. (1999). Proyecto de Enseñanza para la Comprensión.

Pichon-Rivieré, E. (2015) La teoría del aprendizaje. 1968 Grupalista/Biblioteca de Psicología Social Pichoniana.

Vazquez Cabanillas, C. (2004). El Auxiliar del Conductor de Obras. Planificar - Organizar Dirigir - Evaluar. Buenos Aires: Alsina. 